\title{
PROFILE OF COVID -19 PATIENTS AT A DEDICATED COVID HEALTH CENTRE (DCHC) IN SUBURBS OF A METROPOLITAN CITY IN MUMBAI INDIA -A RETROSPECTIVE STUDY.
}

KEY WORDS: Covid 19 $\mathrm{DCHC}$
Dr Pandya

Jayashri S

Dr.Ajit Nair

Dr Andrade

Neelam

Dr Ambre Sagar

Ramesh*

Dr. Sahoo

Durgesh $\mathbf{P}$
MS, FICS, Professor department of General Surgery TNMC Mumbai

MDS periodontology and oral implantology

Dean NESCO, Dean Nair Dental College Professor department of oral and maxillo facial surgery Nair hospital Dental college Mumbai

MBBS, MS, FIOS, FMAS Assistant professor department of general surgery TNMC Mumbai*Corresponding Author

MBBS, MD Assistant professor department of community and family medicine, AIIMS Bibi Nagar Hyderabad

Context: COVID-19 pandemic had over the past months grown to disastrous proportions and had stretched the healthcare facilities across the globe to their limit. Jumbo centres not only helped in increasing the availability of beds for Covid patients, but also helped in de-cluttering the load on tertiary centres. Aim: Clinical and epidemiological profile of patients who received treatment at the MCGM jumbo covid facility at Mumbai. Setting and design: The current study is a retrospective, observational analysis of epidemiological features, clinical manifestations and laboratory and radiographic investigations of patients admitted at the DCHC between June lst 2020 to August 31 st $2020 . \mathbf{M e t h o d s}$ and Material: Patients from June 2020 to August 2020 at DCHC were enrolled in the study. All patients with positive RTPCR, were divided according to symptomatology and classified into Mild, Moderate, Severe. Statistical analysis used: Data was entered in Excel spreadsheet and statistical analysis was done by using SPSS software version 19. Results: Out of 3977 cases, 1620 (40.7\%) cases were mild cases, 1269 (31.9\%) moderate cases and 1088 (27.4\%) severe cases. The age amongst mild cases (50.78 \pm 14.56 years) was higher as compared to moderate (48.23 \pm 15.63 years) and severe cases $(47.18 \pm 15.53$ years $)$ and the difference between all the three groups was statistically significant. Conclusion: DCHC management helped in formulating protocols and with advances in the infrastructure, led to better management with less transfer and helped in preparing for second wave.

\section{INTRODUCTION}

COVID-19 pandemic had over the past months grown to disastrous proportions and had stretched the healthcare facilities across the globe to their limit.[1] The response mounted to the COVID-19 threat has largely been reactive. The lack of a reliable Early Warning, Alert and Response System, inability to mount transparent containment measures, lack of community engagement for self-deferral and isolation, and overdependence on quarantine measures have exposed the fissures in the ability of health systems across the world. However, the emphasis on data sharing, the rapid development and distribution of interim guidance documents by WHO and open-access pre-print sharing of rapidly emerging evidence reflect a paradigm shift in providing a data-driven global-epidemic response. [2]

With increasing number of cases and the rapid spike in bed occupancy in hospitals, the burden on the health care system was compounded. A quick response to this was facilitation of Jumbo centres across the city catering to thousands of patients.

Jumbo centres not only helped in increasing the availability of beds for Covid patients, but also helped in de-cluttering the load on tertiary centres. The profile of patients admitted to DCHC between June 2020 to August 2020 is presented here. The second wave of the pandemic has presented with the different profile, both the profiles can help guide the public health response in India for future waves.

\section{METHODS}

The current study is a retrospective, observational analysis of epidemiological features, clinical manifestations and laboratory and radiographic investigations of patients admitted at the DCHC between June $1^{\text {st }} 2020$ to August $31^{\text {st }}$
2020. The patients admitted at this centre received treatment in accordance to the guidelines issued by MOHFW and underwent laboratory and radiographic investigations to assess disease severity and assist the treatment. ${ }^{[3,4,5]}$ In the first wave of COVID 19 pandemic, there was no proven antiviral therapy effective in controlling the infection, so the measures used during the initial phase were effective infection control, including patient isolation. This formed the main stay, in the primary management. Due to increase in COVID 19 positive cases number there was immediate need for dedicated COVID 19 health care centres. Patients from June 2020 to August 2020 at DCHC were enrolled in the study. All patients with positive RTPCR were recruited in this study. Patients were divided according to symptomatology and classified into Mild, Moderate, Severe, Each was further subdivided into two groups for facilitation of treatment.

Classification according to symptomatology and management-

Mild (Group A, Group B) -

No Symptoms or Mild Symptoms (Fever $\backslash U R T I)$ SPO $2>90 \%$

BLOOD INVESTIGATION-WBC,LDH,D-dimer-Normal

Normal X-ray Chest

Treatment Given- Vit D 1000 IU BD, Vit C 500mg BD, Vit Bcomplex,Tab. Paracetamol

$650 \mathrm{mg}$ TDS for 5 days, Tab. Amoxicillin Clavulanate (Augmentin) 625/Tab. Cefixime 200mg BD, Antitussive.

Moderate (Group C, Group D)-

Symptoms (Fever \URTI\Mild Pneumonia) No sign of severe disease.

SPO2 $>90 \%$ with intermittent $\mathrm{O} 2$ support

BLOOD INVESTIGATION-WBC,LDH,D-dimer-Raised 
$\mathrm{X}$-ray Chest - Mild Pneumonia

Treatment Given- Vit D 1000 IU BD, Vit C 500mg BD, Vit Bcomplex, Tab. Paracetamol 650mg TDS for 5 days, Tab. Amoxicillin Clavulanate (Augmentin)625/Tab. Cefixime 200mg BD, Antitussive, Tab. Favipiravir 1800mg BD on Day 1 followed by $800 \mathrm{mg} \mathrm{BD}$ for the next 6 days.

If sequential fall of SPO2 despite O2 support noticed than Inj. Remdesivir 200mg IV on Day 1 followed by 100mg IV from Day 2 to Day 5/ Tab. Favipiravir (Pyrazine Carboxamide derivative) $1800 \mathrm{mg}$ BD on Day 1 followed by $800 \mathrm{mg}$ BD for the next 6 days, Inj. Ceftriaxone lg IV BD for 5 days, Inj. LMWH (low molecular weight heparin) $0.4 \mathrm{~s} / \mathrm{c}$ OD, Inj. MPS (Methylprednisolone) 40mg BD/ Inj. Dexa (Dexamethasone) 8mg BD IV (if patient still symptomatic at Day 5 therapy continued for another 5 days)

\section{Severe (Group E) -}

Symptoms (Fever \URTI $\backslash$ Pneumonia) Signs of severe disease. SPO $2<90 \%$ On continuous O2 support

RR->30 $\backslash$ Min

BLOOD INVESTIGATION-WBC,LDH,D-dimer-Raised

$\mathrm{X}$-ray Chest - Pneumonia

Treatment Given- Vit D 1000 IU BD, Vit C 500mg BD, Vit Bcomplex,Tab.Paracetamol

650mg TDS for 5 days, Tab. Amoxicillin Clavulanate (Augmentin) 625/Tab. Cefixime 200mg BD, Antitussive, Tab. Favipiravir (Pyrazine Carboxamide derivative) 1800mg BD on Day 1 followed by $800 \mathrm{mg}$ BD for the next 6 days.

Inj. Remdesivir 200mg IV on Day 1 followed by $100 \mathrm{mg}$ IV from Day 2 to Day 5, Inj. Ceftriaxone lg IV BD for 5 days, Inj . LMWH (low molecular weight heparin) $0.4 \mathrm{~s} / \mathrm{c}$ OD, Inj. MPS (Methylprednisolone) 40mg BD/Inj. Dexa (Dexamethasone) $8 \mathrm{mg}$ BD IV (if patient symptomatic at Day 5 , therapy continued for additional 5 days).

\section{Criteria for transfer-}

- Progression from moderate to severe disease and further deterioration.

- Lack of facilities like ICU, non-avability of essential injectables.

- Unwillingness of patients or relative to stay in DCHC.

\section{Data collection-}

Clinical and laboratory data collected, including age, sex, comorbidities, symptoms, RT PCR for detection of Covid19, duration of stay will be statistically analysed. Data was collected and verified and all patients with positive RTPCR enrolled. Basic information (Gender, age, comorbidities, symptoms like fever, cough, loss of smell, body pain, loss of taste, diarrhoea) white blood cells (WBC) counts, Lactate dehydrogenase (LDH) count, (D-dimer) fibrin degradation product levels, CXR were collected and analysed.

\section{RESULT-}

Out of 3977 cases, 1620 (40.7\%) cases were mild cases, 1269 $(31.9 \%)$ moderate cases and $1088(27.4 \%)$ severe cases. The age amongst mild cases ( $50.78 \pm 14.56$ years) was higher as compared to moderate ( $48.23 \pm 15.63$ years) and severe cases $(47.18 \pm 15.53$ years) and the difference between all the three groups was statistically significant. Similarly, SPO2 level was higher amongst mild cases (92.72 $\pm 1.28 \%)$ as compared to moderate $(91.40 \pm 1.79 \%)$ and severe cases $(88.00 \pm 0.00 \%)$ and all the three groups were statistically significant. Length of stay was highest among severe cases $(4.04 \pm 1.71$ days $)$ followed by moderate ( $3.71 \pm 9.33$ days) and mild cases (2.94 \pm 14.40 days). The difference of length of stay between mild and severe cases was statistically significant while there was no difference between mild and moderate $(p$ value $=0.054)$ or between moderate and severe cases ( $p$ value $=0.450$ ).
Amongst all the three groups, males were higher (mild = $62.2 \%$, moderate $=64.1 \%$, severe $=65.2 \%$ ) as compared to females and the difference amongst all the three groups was not statistically significant. Presence of comorbidities was highest amongst severe group (36.9\%) as followed by moderate $(33.5 \%)$ and mild were $(28.3 \%)$ and the difference was significant amongst all the three groups. Severe cases are transferred more $(36.6 \%)$ followed by moderate cases $(6.7 \%)$ and mild cases $(0.6 \%)$ and the difference was statistically significant amongst all the three groups.

All the cases in severe groups (100.0\%) had abnormal TLC (either raised or low) followed by mild cases (10.4\%) and moderate cases $(6.5 \%)$ and the difference amongst all the three groups was statistically significant. LDH level was maximumly raised amongst mild cases (14.4\%) followed by severe cases (5.4\%) and moderate cases (2.0\%). D Dimer was highest raised amongst mild cases $(91.0 \%)$ followed by moderate $(64.0 \%)$ and severe cases $(15.7 \%)$, but the difference was significant amongst mild and severe cases but not amongst moderate cases. Pneumonia was present almost similarly amongst both moderate cases (49.9\%) and mild cases $(48.3 \%)$ as compared to severe cases $(8.5 \%)$ and the difference was statistically significant amongst all the three groups.

Table 1: Comparison of age, SPO2 and length of stay between mild moderate and severe groups.

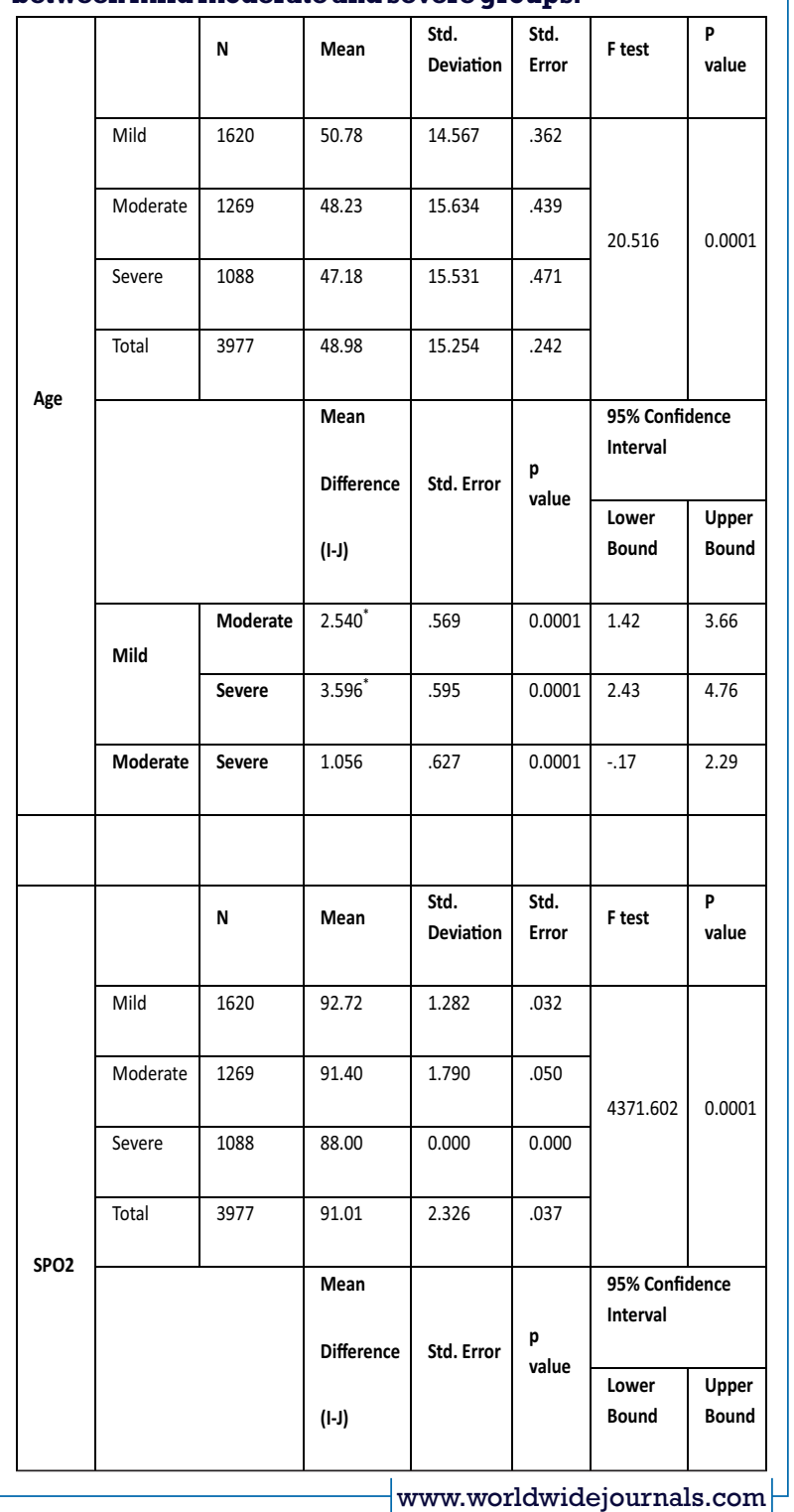


PARIPEX - INDIAN JOURNAL OF RESEARCH | Volume - 10 | Issue - 08 |August - 2021 | PRINT ISSN No. 2250 - 1991 | DOI : $10.36106 /$ paripex

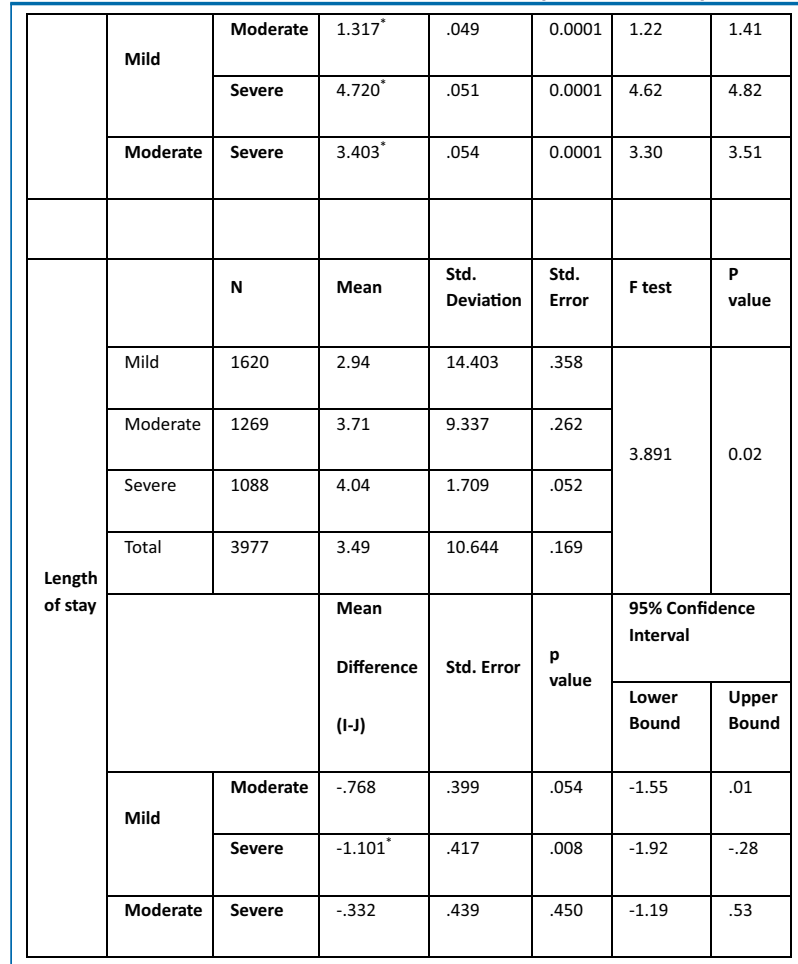

Table 2: Comparison of gender, comorbidities, and transfer details between mild moderate and severe groups

\begin{tabular}{|c|c|c|c|c|c|}
\hline & & & Group & & \\
\hline & & & Mild & Moderate & Severe \\
\hline & & Count & 613 & 455 & 379 \\
\hline & & $\%$ & $37.8 \%$ & $35.9 \%$ & $34.8 \%$ \\
\hline & & Count & 1007 & 814 & 709 \\
\hline & Male & & & & \\
\hline & & $\%$ & $62.2 \%$ & $64.1 \%$ & $65.2 \%$ \\
\hline Chi-square & & & 2.501 & 0.225 & 1.554 \\
\hline$p$ value & & & 0.1138 & 0.635 & 0.213 \\
\hline & & Count & 459 & 425 & 401 \\
\hline Co- & & $\%$ & $28.3 \%$ & $33.5 \%$ & $36.9 \%$ \\
\hline moriagities & & Count & 1161 & 844 & 687 \\
\hline & Absent & & & & \\
\hline & & $\%$ & $71.7 \%$ & $66.5 \%$ & $63.1 \%$ \\
\hline Chi-square & & & 19.77 & 14.74 & 14.15 \\
\hline$p$ value & & & 0.0001 & 0.0001 & 0.0001 \\
\hline & & Count & 10 & 85 & 398 \\
\hline ansfer & & $\%$ & $0.6 \%$ & $6.7 \%$ & $36.6 \%$ \\
\hline Detall & Not & Count & 1610 & 1184 & 690 \\
\hline & & $\%$ & $99.4 \%$ & $93.3 \%$ & $63.4 \%$ \\
\hline Chi-square & & & 349.2 & 55.72 & 806.7 \\
\hline$p$ value & & & 0.0001 & 0.0001 & 0.0001 \\
\hline
\end{tabular}

Table 3: Comparison of investigation reports between mild moderate and severe groups

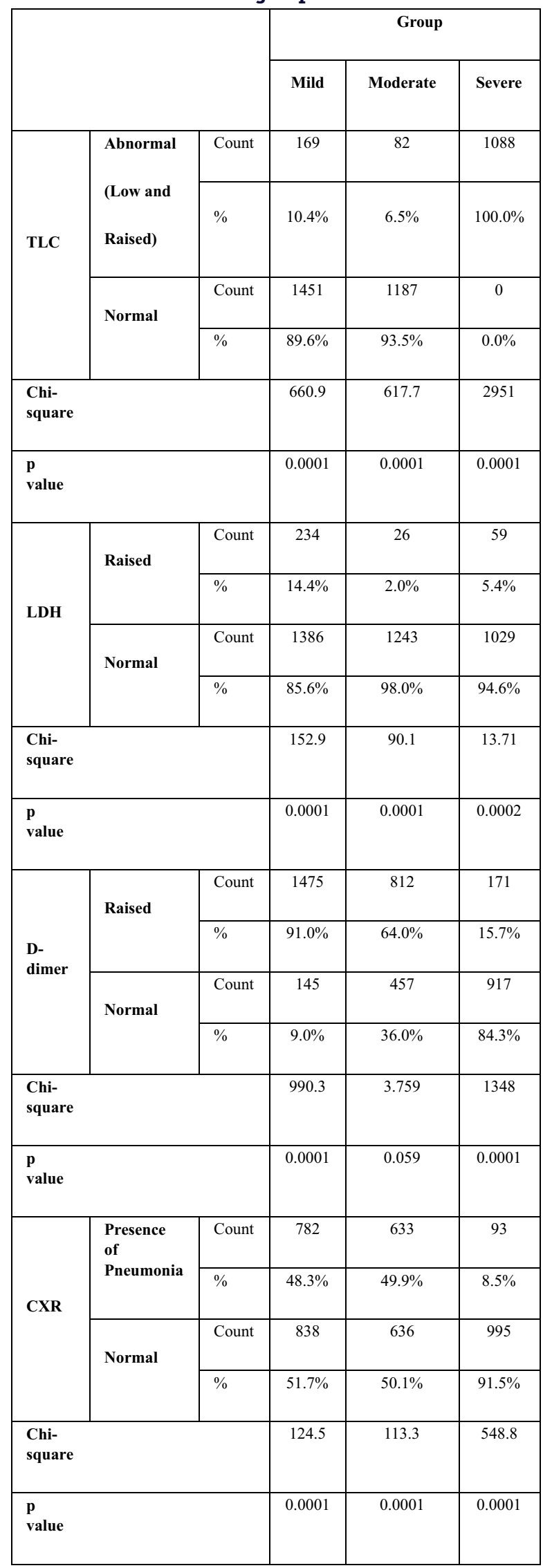

\section{DISCUSSION-}

In Mumbai last year in the month of august 2020 total of cases 
reported were $1,21,027$ and DCHC, Mumbai city and suburban areas reported 1,109 and 3,324 fresh cases of COVID-19, respectively till $31^{\text {st }}$ august.

We studied the profile and clinical characteristics and outcome of 3977 COVID 19 patients in DCHC.

In our study, primary symptom profile was fever and cough, loss of taste, body pain, loss of smell.

In our study $11 \%$ of cases were transferred which is similar to study done by XWang et $\mathrm{al}^{[8]}$.

COVID 19 is highly communicable viral infection associated with overactive host immune response resulting in pneumonia.

In our study the duration of symptoms lasted from 4-7 days with complication setting at 7 day at an average which is similar to the, a study done by C.V.Verma et $\mathrm{al}^{[6]}$ which stated duration of symptoms as approximately 4-10 days with complication setting in between 4-19 days .

In our study, the age group of cases involved in each group with comorbidities ranged from $39-50$ years of age.

In our study there was some atypical symptoms like, loss of taste, loss of smell, diarrhoea noted, which is similar to Study done by Wang et al where atypical symptoms, like diarrhoea were noted in $2-10 \%$ of cases, and other atypical symptoms like loss of taste, loss of smell ranged from 30 to $20 \%$ of cases ${ }^{[7,8]}$

In our study abnormal TLC (either raised or low) was seen $(100.0 \%)$ in severe groups followed by mild cases $(10.4 \%)$ and moderate cases $(6.5 \%)$ and the difference amongst all the three groups was statistically significant. LDH level was maximum raised amongst mild cases (14.4\%) followed by severe cases (5.4\%) and moderate cases (2.0\%).D Dimer was raised more amongst mild cases $(91.0 \%)$ followed by moderate $(64.0 \%)$ and severe cases $(15.7 \%)$ but the difference was significant amongst mild and severe cases but not amongst moderate cases.

In our study the number of mild cases were $40.73 \%$, moderate cases were $31.9 \%$, and severe cases were $27.35 \%$.

DCHC was a single centre built on war footing to tackle the pandemic. During the initial phases, routine investigations and radiology of the chest was done. With the increase in number of cases, management was done according to the symptom complex and set transfer criteria. Due to surge, there was lack of essential medicine due to increase demand. DCHC had no CT scan facilities also ICU facilities were absent this led to increase in the rate of transfer in initial phases.

\section{CONCLUSIONS}

In conclusion DCHC management helped in formulate protocols and with advances in the infrastructure, led to better management with less transfer. Not only the personnel learnt, but also, administration could also set protocols. This helped us to prepared us for future waves.

\section{REFERENCES:}

1. World Health Organization. 2019-nCoV Situation Report. Indian J Med Res 151,February \& March 2020, pp 147159

2. Seith Bhalla, Ashu \& Jana, Manisha \& Naranje, Priyanka \& Manchanda, Smita. (2020). Role of Chest Radiographs during COVID-19 Pandemic. Annals of the National Academy of Medical Sciences (India). 10.1055/s-0040-1 714158.

3. Centers for Disease Control and Prevention. Real-time RT-PCR panel for detection 2019-novel coronavirus. February 4,2020

4. Centers for Disease Control and Prevention. Interim guidelines for collecting, handling, and testing clinical specimens from persons for coronavirus disease 2019 (COVID-19). 2020

5. WHO-Global Surveillance for COVID-19 Disease Caused by Human Infection with the 2019 Novel Coronavirus Interim Guidance: 27 February 2020. Available from:https://www.who.int/publications-detail/globalsurveillance-for-human-infection-with- novel- coronavirus-(2019-ncov).
6. C.V.Verma et al Physiotherapy management of COVID-19 patients in acute set up of government tertiary care hospital: A case series September 2020Journal of Society of Indian Physiotherapists 4(2):101-106

7. X.Wang et al Clinical characteristics of non-critically ill patients with novel coronavirus infection (COVID-19) in a Fangcang Hospital

8. Wang D., Hu B., Hu C., Zhu F., Liu X., Zhang J. Clinical characteristics of 138 hospitalized patients with 2019 novel coronavirus-infected pneumonia in Wuhan, China.JAMA. 2020. 